Medicamentos contra la obesidad y el sobrepeso: resultados de un metaanálisis

La obesidad y el sobrepeso son trastornos crónicos de alta prevalencia que afectan en la actualidad a más de mil millones de personas en todo el mundo y están asociados con la mortalidad prematura, diversas enfermedades crónicas y un mayor uso de los servicios de salud. Aunque según directivas publicadas recientemente, el tratamiento inicial contra la obesidad se debe basar en modificaciones del estilo de vida, se recomienda aplicar tratamientos con medicamentos en pacientes con índice de masa corporal (IMC) $\geq 30$ o con valores del IMC entre 27 y 29,9 si se observan complicaciones debido a la obesidad. Entre los medicamentos aprobados para el tratamiento prolongado contra la obesidad (un año o más) se encuentran el orlistat, un inhibidor de la lipasa gastrointestinal; la sibutramina, un inhibidor de la recaptura de monoaminas de acción centralizada; y el rimonabant, un antagonista de los receptores de endocannabinoides.

El uso de medicamentos contra la obesidad es frecuente. Se estima que las ventas de estos medicamentos en 2005 alcanzaron 1,2 millones de dólares estadounidenses. Como la pérdida de peso mediante intervenciones basadas en cambios del estilo de vida es limitada y de corta duración - debido a las recidivas y al enlentecimiento compensatorio del metabolismo- y la prevalencía de la obesidad va en aumento, el empleo de tratamientos con medicamentos contra la obesidad debe aumentar en el futuro cercano.

En este trabajo se presentan los resultados de una revisión sistemática y un metaanálisis dirigidos a cuantificar la eficacia de los medicamentos más frecuentemente empleados contra la obesidad, así como a caracterizar sus efectos secundarios. La revisión sistemática actualizada recientemente por Cochrane Collaboration se basó en la información publicada entre diciembre de 2002 y diciembre de 2006 e indexada por Medline, Embase, el registro de ensayos controlados de Cochrane y el registro de ensayos controlados de Current Science y en las referencias de los artículos identificados. Se encontraron 30 ensayos que tenían una duración de 1 a 4 años: 16 emplearon orlistat ( $n=10631$ participantes), 10 utilizaron sibutramina ( $n=2$ 623) y 4 usaron rimonabant $(n=6365)$. De ellos, 14 ensayos eran nuevos y 16 se habían identificados previamente.
Se observó que todos los medicamentos dieron como resultado una pérdida promedio de peso de menos de $5 \mathrm{~kg}$ en comparación con el placebo. No se encontraron datos sobre el efecto de estos medicamentos en la mortalidad o la morbilidad cardiovascular. Los estudios sobre el mantenimiento del peso corporal con cada uno de los medicamentos analizados demostraron que la recuperación del peso fue similar en el grupo tratado y en el de placebo, de manera que se mantuvieron las diferencias originales en el peso de ambos grupos. No se encontraron efectos diferenciadores en cuanto a las reacciones secundarias. Estos resultados confirman hallazgos anteriores, pero definen con mayor precisión los efectos a largo plazo de estos medicamentos con respecto a la pérdida de peso y sus efectos secundarios.

A pesar de algunas limitaciones del trabajo, se observó que en los estudios en los que participaron pacientes diabéticos, la pérdida de peso con orlistat y rimonabant fue ligeramente menor que con sibutramina. No obstante, tanto el orlistat como el rimonabant redujeron los niveles de glucemia en los pacientes diabéticos, mientras que la sibutramina no mejoró este parámetro. Aunque no se conocen las causas de este importante hallazgo, un factor que puede influir en esta reducción de los niveles de glucemia con el rimonabant es el aumento en los niveles de adiponectina. Se requieren investigaciones adicionales, preferentemente mediante ensayos clínicos con los tres medicamentos, para establecer las conclusiones definitivas.

A pesar de que los pacientes se seleccionaron por su adhesión y su tolerancia a los tratamientos, las tasas de abandono fueron elevadas, lo que comprometió la validez interna de muchos de los estudios analizados. La falta de adhesión al tratamiento parece constituir el principal factor que limita la eficacia y la efectividad de los medicamentos contra la obesidad.

Con relación a la sibutramina, es preocupante el aumento observado en la tensión arterial de los pacientes, por lo que se debe monitorear este signo vital durante el tratamiento. También se debe vigilar el estado emocional de los pacientes que emplean rimonabant, especialmente en personas con trastornos psiquiátricos.

La decisión de prescribir un medicamento para bajar de peso debe basarse en un cuidadoso balance entre los riesgos y los beneficios del trata- 
miento específico que se propone. Los medicamentos empleados actualmente para este fin son costosos y tienen diversos efectos secundarios. A falta de datos definitivos que demuestren que un medicamento en particular es más efectivo que los otros, se debe establecer el tratamiento inicial de acuerdo con las preferencias del paciente, los costos locales del medicamento, su disponibilidad y cobertura por las compañías aseguradoras de servicios de salud, los efectos colaterales del medicamento y su relación con otras enfermedades que pueda presentar el paciente. La importancia clínica de la reducción observada en la incidencia de la diabetes al emplear orlistat no está clara y se requieren investigaciones adicionales para determinar su verdadero efecto preventivo. (Rucker D, Padwal R, Li SK, Curioni $\mathrm{C}$, Lau DCW. Long term pharmacotherapy for obesity and overweight: updated meta-analysis. $\mathrm{Br}$ Med J. 2007;335:1194-9).

\section{Características de la malaria por Plasmodium falciparum y P. vivax en la Amazonía brasileña}

Los estudios sobre la prevalencia de la malaria en el estado de Amazonas y la ciudad de Manaos, Brasil, indican que hay un aumento en el porcentaje de pacientes hospitalizados por infección con Plasmodium vivax y un incremento en el número de casos de malaria causados por este parásito. Ese aumento en el número de pacientes hospitalizados está asociado con una mayor frecuencia de casos graves de malaria por P. vivax. El estado Amazonas abarca una gran parte de la Amazonía brasileña, donde la malaria ha estado controlada pero nunca erradicada. Desde la década de 1980 se ha observado una reemergencia de la malaria, que parece coincidir con los cambios en las políticas de control de esta enfermedad relacionados con la conclusión de la Campaña de Erradicación de la Malaria.

En este trabajo se analiza la epidemiología de la malaria en el estado de Amazonas y la ciudad de Manaos entre 1980 y 2006 y se describen las tasas generales, la prevalencia y las tasas de admisión de pacientes con malaria, tanto por P. falciparum como por $P$. vivax. Para ello se tomaron el número total de casos de malaria y la población del estado de Amazonas a partir de las bases de datos del Ministerio de Salud de Brasil, la Fundación Nacional de Salud y la Secretaría de Vigilancia Sanitaria. Los datos sobre el diagnóstico y el tratamiento de la malaria de la Fundación de Medicina Tropical del estado de Amazonas (FMT-AM) se tomaron directamente de los registros de malaria (en los años previos a la publicación de los informes trimestrales de la Fundación) y de los informes trimestrales (en los años posteriores).
El número de casos de malaria en Amazonas muestra un incremento irregular a partir de 1988, con un pico en 1999 seguido de una disminución en el año 2001 y un nuevo aumentó en los años siguientes. Se observó una disminución en el número de casos en 2006, aunque no se cuenta con las cifras definitivas. El número total de casos de malaria diagnosticados en la FMT-AM fluctuó de manera similar a lo observado en el estado en su conjunto.

En 1989, 264 (20\%) de los pacientes diagnosticados con malaria por $P$. falciparum ingresaron a hospitales, mientras que solo $26(0,85 \%)$ de los infectados por $P$. vivax necesitaron hospitalización. Aunque $P$. falciparum continuó siendo la principal causa de hospitalización, se observó un incremento significativo en el número de casos de malaria por P. vivax hospitalizados entre 1989 y 1996 (0,59\%; desviación estándar [DE]: 0,18); esa cifra aumentó a $1,91 \%(\mathrm{DE}=0,74)$ entre 1997 y 2006. Este aumento relativo en el número de casos de malaria por $P$. vivax que requirieron hospitalización para su tratamiento no guarda proporción con el incremento en el número de enfermos y la frecuencia relativa de casos de malaria causados por $P$. vivax con respecto a los causados por P. falciparum.

Las características epidemiológicas de los casos de malaria registrados en el estado de Amazonas y la ciudad de Manaos en los últimos años demuestran la emergencia de una forma de malaria más grave causada por $P$. vivax. Si se asume que los pacientes que requirieron hospitalización se encontraban más enfermos que los que no lo requirieron, se puede concluir que la transmisión de la malaria en esta región ha sido continua, pero su intensidad ha variado. Si bien $P$. vivax ha sido la causa principal de malaria, el número de pacientes con infección por este parásito que han requerido hospitalización ha aumentado significativamente en los años recientes. Los cambios en las operaciones de control han estado asociados con la aparición de la malaria en las principales zonas urbanas de la cuenca del Amazonas. En Manaos, esto puede haber influido en las manifestaciones y la gravedad de la enfermedad por $P$. vivax, pero no parece haber influido en la gravedad de la infección por $P$. falciparum, tal vez debido a la política actual de diagnóstico y tratamiento tempranos, que ha influido más en el control de los casos de malaria causada por $P$. falciparum que en los asociados con P. vivax.

Aunque la definición de caso y los criterios de hospitalización empleados en la FMT-AM fueron idénticos durante todo el período de estudio, tanto para la infección por P. vivax como por P. falciparum, la probabilidad de hospitalización de los pacientes diagnosticados con malaria por $P$. vivax aumentó significativamente después de 1996, mientras que el porcentaje de hospitalizaciones por malaria cau- 